\title{
On the Geometric Control of Internal Forces in Power Grasps
}

\author{
D. Prattichizzo ${ }^{\dagger \ddagger}$ \\ P. Mercorelli $\ddagger$ \\ A. $\operatorname{Bicchi}^{\dagger}$ \\ A. Vicino \\ †Dipartimento di Sistemi Elettrici e Automazione, Università di Pisa \\ via Diotisalvi, 2, 56100 Pisa, Italia. prattichizzo@dsea.unipi.it, bicchi@dsea.unipi.it \\ $\ddagger^{\ddagger}$ Dipartimento di Ingegneria dell'Informazione, Università di Siena \\ via Roma, 56, 53100 Siena, Italia. mercorelli@unisi.it, vicino@unisi.it
}

\begin{abstract}
The geometric approach to the control of internal forces for robotic grasping is explored. Since the manipulation of deformable objects is a frequently occurrence (medical applications, manipulation of rubber and plastic in industry), manipulation systems with significant contact elasticity are studied. The presence of non-negligible compliance at the contacts, implies that the object dynamics cannot be neglected when attempting to control internal forces without affecting the object position.

A geometric approach to derive a control law decoupling the internal force control action from the object dynamics is proposed.
\end{abstract}

\section{Introduction}

In robotic literature terms as "whole-arm" and "enveloping" or "power" grasps are representative of all those situations where the manipulator touches the gripped object with its own inner parts and not only with the end-effectors, $c f .[11,4,12]$ and references therein.

In order to execute power grasps, robotic manipulators must be equipped with appropriate hardware. In fact, inner parts must be sensorized as well as fingertips. As a consequence the complexity of the analysis and the control synthesis increases consistently. For instance, particular attention must be devoted to specify consistent control goals. In [8] it was shown that in general a power grasp cannot move the manipulated object in any direction of its configuration space.

From an analytical point of view, power grasps involves the presence of kinematical deficiency $[9,10]$. In other words, in power grasps usually at least one of the links touching the object possesses less degrees-of-freedom than those necessary to move its contact point in arbitrary directions. Thus there exists a non-zero null

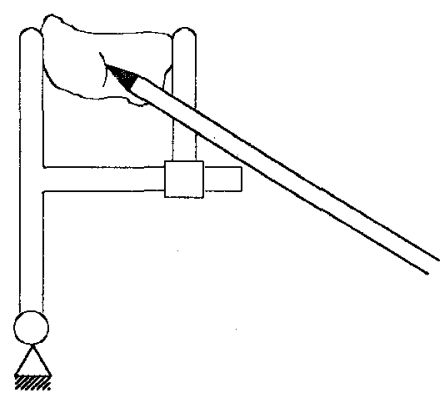

Figure 1: Micro manipulation of internal tissues in surgery or laparoscopy.

space of the Jacobian transpose, $\operatorname{ker}\left(\mathbf{J}^{\mathbf{T}}\right) \neq \mathbf{0}$, and the manipulation system is said kinematically defective.

This work is focused on the control of internal forces for kinematically defective manipulation systems with non-negligible contact elasticity. In [9] it was shown that such a class of manipulators is enough general to include a wide variety of manipulators and cooperating robots.

Internal forces are defined as those having no action on the object dynamics. They are self-balanced forces and belong to the null space of the grasp matrix (cf. e.g. [9]). Internal forces play a key role in controlling the grasp.

Frequently, in advanced robotics, contact (object and/or fingertips) elasticity cannot be neglected. In -industry this occurs whenever there is a need of assembling and manipulating non-rigid (rubber or plastic) parts. In medical applications, as the micro manipulation of tissues in surgery or in laparoscopy, for instance there could be the need of squeezing the tissue part of the patient's organ in order to exert a cutting action, see fig. 1. Moreover note that modelling contact elasticity is mandatory not only for deformable manipulated parts but also for soft fingertips as those of Akella and 


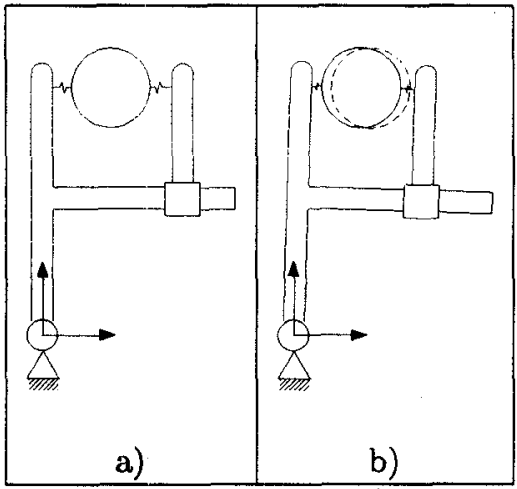

Figure 2: Deformable contacts: squeezing action of the prismatic joint.

Cutkoski [1].

If the manipulation system can be modelled by rigidbody contacts, usually the object dynamics is intentionally ignored to synthesize the internal-force controller. On the other hand, for the aforementioned examples of advanced manipulation tasks, the object dynamics cannot be disregarded at all in synthesizing the internal-force controller. As an example consider the manipulation system of fig. 1 and model the complaint contacts with visco-elastic lumped parameters as depicted in fig.2-a). A control policy ignoring object dynamics, for instance a force step on the prismatic joint, squeezes the manipulated object but an undesired and dangerous transient motion of the object arises.

In this paper a state-feedback controller that perfectly decouples the internal force control loop from the object dynamics is proposed.

\section{Background}

This paper is related to previous works by the authors $[8,9,10]$ where the linearized dynamics of manipulators with general kinematics and their system-theoretic structural properties were investigated. Some of these results along with notation are here summarized for the reader's convenience.

Let $\mathbf{q} \in \mathbf{R}^{\mathbf{q}}$ be the vector of joint positions, $\tau \in \mathbb{R}^{q}$ the vector of joint generalized forces, $\mathbf{u} \in \mathbf{R}^{\mathbf{d}}$ the vector locally describing the position and the orientation of a frame attached to the object and finally $\mathbf{w} \in \mathbf{R}^{\mathbf{d}}$ the vector of forces and torques resultant from external forces acting on the object.

Let further introduce the vector $t \in \mathbb{R}^{\mathbf{t}}$ whose components include contact forces and torques. We assume that contact forces arise from a lumped-parameter model of visco-elastic phenomena at the contacts, summarized by a stiffness matrix $\mathbf{K}$ and a damping matrix
B. Note that the lumped parameter model of contact interactions makes the identification procedure feasible with a reasonable amount of computations.

The Jacobian $\mathbf{J}$ and the grasp matrix $\mathbf{G}$ are defined as the linear maps relating the velocities of the contact points on the links and on the object, to the joint and object velocities, respectively, cf. [5].

The linear approximation of the manipulation system dynamics in the neighbourhood of such an equilibrium configuration, $(\mathbf{q}, \mathbf{u}, \dot{\mathbf{q}}, \dot{\mathbf{u}}, \tau, \mathbf{t})=\left(\mathbf{q}_{\mathbf{o}}, \mathbf{u}_{\mathbf{o}}, \mathbf{0}, \mathbf{0}, \tau_{\mathbf{0}}, \mathbf{t}_{\mathbf{o}}\right)$, can be written as

$$
\dot{\mathbf{x}}=\mathbf{A x}+\mathbf{B}_{\tau} \tau^{\prime}+\mathbf{B}_{\mathbf{w}} \mathbf{w}^{\prime},
$$

where state and input vectors are defined as the departures from the reference equilibrium configuration: $\mathbf{x}=\left[\left(\mathbf{q}-\mathbf{q}_{\mathbf{o}}\right)^{\mathbf{T}}\left(\mathbf{u}-\mathbf{u}_{\mathbf{o}}\right)^{\mathbf{T}} \dot{\mathbf{q}}^{\mathbf{T}} \dot{\mathbf{u}}^{\mathbf{T}}\right]^{\mathbf{T}}, \tau^{\prime}=\tau-\mathbf{J}^{\mathbf{T}} \mathbf{t}_{\mathbf{o}}$ $\mathbf{w}^{\prime}=\mathbf{w}+\mathbf{G t}_{\mathbf{o}}$, and

$\mathbf{A}=\left[\begin{array}{cc}\mathbf{0} & \mathbf{I} \\ \mathbf{L}_{\mathbf{k}} & \mathbf{L}_{\mathbf{b}}\end{array}\right] ; \mathbf{B}_{\tau}=\left[\begin{array}{c}\mathbf{0} \\ \mathbf{0} \\ \mathbf{M}_{\mathbf{h}}^{-1} \\ \mathbf{0}\end{array}\right] ; \quad \mathbf{B}_{\mathbf{w}}=\left[\begin{array}{c}\mathbf{0} \\ \mathbf{0} \\ \mathbf{0} \\ \mathbf{M}_{\mathbf{0}}^{-1}\end{array}\right]$, being $\mathbf{M}_{\mathbf{h}}$ and $\mathbf{M}_{\mathbf{o}}$ the inertia matrices of the manipulator and the object, respectively. To simplify notation, the prime in $\tau^{\prime}$ and $\mathbf{w}^{\prime}$ will be henceforth omitted.

Assuming that local variations of gravity forces on system dynamics, of the Jacobian and of the grasp matrices are negligible for small displacements $\delta \mathbf{u}, \delta \mathbf{q}$, simple expressions are obtained, [8] for

$$
\mathbf{L}_{\mathbf{k}}=-\mathbf{M}^{-\mathbf{1}} \mathbf{P}_{\mathbf{k}}, \quad \mathbf{L}_{\mathbf{b}}=-\mathbf{M}^{-\mathbf{1}} \mathbf{P}_{\mathbf{b}}
$$

where $\mathbf{M}=\operatorname{diag}\left(\mathbf{M}_{\mathbf{h}}, \mathbf{M}_{\mathbf{o}}\right), \mathbf{P}_{\mathbf{k}}=\mathbf{S}^{\mathbf{T}} \mathbf{K S}, \mathbf{P}_{\mathbf{b}}=$ $\mathbf{S}^{\mathbf{T}} \mathbf{B S}$, and $\mathbf{S}=\left[\mathbf{J}-\mathbf{G}^{\mathbf{T}}\right]$.

The framework throughout is the geometric approach to the structural synthesis of multivariable systems. Such approach, pioneered by Basile and Marro $[2,3]$ and Wonham [13], has been used to obtain structural geometric decoupling conditions in the state space.

\section{Internal forces as controlled outputs}

The control of contact forces $t$ is a fundamental part of the manipulation control problem. Contact forces allows the manipulator to maintain the grasp, rejecting external disturbance $\mathbf{w}$ and controlling the object motion.

Consider the departures of contact force vector $\mathbf{t}$ from the reference equilibrium $t_{\mathbf{o}}$. Let $t^{\prime}$ (henceforth $t$ ) be its first order approximation which in [9] was computed as an output of the linearized model (1): $\mathbf{t}=\mathbf{C}_{t} \mathbf{x}$ where $\mathbf{C}_{\mathbf{t}}=\left[\mathbf{K J}-\mathbf{K G}^{\mathbf{T}} \mathbf{B J}-\mathbf{B G}^{\mathbf{T}}\right]$.

Assume that stiffness matrix $\mathbf{K}$ and damping matrix $\mathbf{B}$ are proportional, this is customary in mechanical vibration analysis [6]. It follows that $\operatorname{im}(\mathbf{K})=\mathrm{im}(\mathbf{B})$ 
and that all the geometric results of this paper can be expressed only as functions of $\mathbf{K}$.

In [4] it was shown that, in kinematically defective grasps, not all the internal forces are controllable. Thus an analysis of their reachable set is needed in order to specify consistent control goals. In [10] the authors, starting from the linearized dynamics, define the reachable internal forces subspace $\mathcal{R}_{t i, \tau}$ as the intersection between the reachable subspace of all the contact force and the null space of the grasp matrix:

$$
\mathcal{R}_{t i, \tau}=\mathcal{R}_{t, \tau} \cap \operatorname{ker}(\mathbf{G}) .
$$

Moreover, in terms of column spaces, they showed that

$$
\begin{aligned}
& \mathcal{R}_{t i, \tau}=\operatorname{im}\left(\mathbf{P}_{\mathbf{N G}} \mathbf{C}_{\mathbf{t}}\right)=\mathrm{im}\left(\mathbf{P}_{\mathbf{N G}} \mathbf{K J}\right) \\
& \text { where } \mathbf{P}_{\mathbf{N G}}=\mathbf{I}-\mathbf{K G}^{\mathbf{T}}\left(\mathbf{G K G} \mathbf{K}^{\mathbf{T}}\right)^{-\mathbf{1}} \mathbf{G}
\end{aligned}
$$

According to this result, the subspace of reachable internal forces is obtained by the projector $\mathbf{P}_{\mathbf{N G}}$ (on the null space of $\mathbf{G}$ ) acting on the column space of $\mathbf{C}_{t}$.

Finally, the regulated force output $\mathbf{e}_{\mathbf{t i}}$ is chosen as the projection of the force vector $t$ onto the null space of $\mathbf{G}$, i.e. the reachable internal contact forces:

$$
\begin{aligned}
& \mathbf{e}_{\mathbf{t i}}=\mathbf{E}_{\mathrm{ti}} \mathbf{x} ; \text { where } \mathbf{E}_{\mathrm{ti}}=\mathbf{P}_{\mathbf{N G}} \mathbf{C}_{\mathbf{t}}=\left[\begin{array}{llll}
\mathbf{Q} & 0 & \mathbf{Q} & 0
\end{array}\right] \\
& \text { and } \mathbf{Q}=\left(\mathbf{I}-\mathbf{K G}^{\mathbf{T}}\left(\mathbf{G K G}^{\mathbf{T}}\right)^{-\mathbf{1}} \mathbf{G}\right) \mathbf{K J} \text {. }
\end{aligned}
$$

It might be worthwhile to emphasize that

$$
\mathcal{R}_{t i, \tau}=\operatorname{im}\left(\mathbf{E}_{\mathbf{t i}}\right)
$$

\section{Rigid-body object motions}

Rigid-body kinematics are of particular interest in the control of manipulation systems. They do not involve visco-elastic deformations of bodies, thus they can be regarded as low-energy motions. Rigid-body kinematics represent the easiest way to move the object.

Rigid-body kinematics have been studied in a quasistatic setting in [5] and in terms of unobservable subspaces from contact forces in [8]. In both cases rigid kinematics were described by a matrix $\Gamma$ whose columns form a basis for $\operatorname{ker}\left[\mathbf{J}-\mathbf{G}^{\mathbf{T}}\right]=\mathrm{im}(\boldsymbol{\Gamma})$ where

$$
\begin{aligned}
& \Gamma=\left[\begin{array}{ccc}
\boldsymbol{\Gamma}_{r} & \boldsymbol{\Gamma}_{q c} & 0 \\
0 & \Gamma_{u c} & \Gamma_{i}
\end{array}\right] \\
& \text { and } \mathbf{J} \Gamma_{\mathbf{q c}}=\mathbf{G}^{\mathbf{T}} \boldsymbol{\Gamma}_{\mathbf{u c}}
\end{aligned}
$$

being $\Gamma_{r}$ a basis matrix (b.m.) of the subspace of redundant manipulator motions $\operatorname{ker}(J), \Gamma_{i}$ a b.m. of the subspace of indeterminate object motions $\operatorname{ker}\left(\mathbf{G}^{\mathbf{T}}\right)$, and $\Gamma_{q c}$ and $\Gamma_{u c}$ conformal partitions of a complementary basis matrix.

The column space of $\Gamma_{c}=\left[\begin{array}{l}\Gamma_{q c} \\ \Gamma_{u c}\end{array}\right]$ consists of coordinated rigid-body motions of the mechanism, for
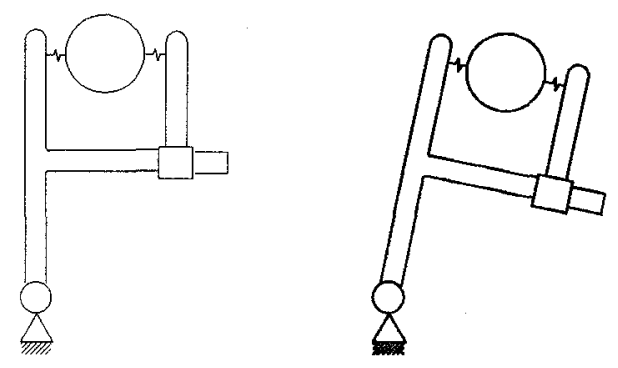

Figure 3: Coordinate rigid-body motion.

the manipulator $\left(\boldsymbol{\Gamma}_{q c}\right)$ and the object $\left(\boldsymbol{\Gamma}_{u c}\right)$ components. Physically rigid-body displacements do not involve variation of contact forces. Fig. 3 shows such subspaces for the simple device of fig. 1 .

In [8], it has been shown that rigid-body motions are reachable, i.e. they belong to the space of reachability of linear system (1) with input vector of joint generalized forces $\tau$. Note that the rigid-body subspace is only a subspace of the reproducible one which also contains motions due to deformations of elastic elements in the model.

The rigid-body object displacements $\mathbf{e}_{\mathbf{u c}}$ are described by the projection, $\Gamma_{u c}^{P}$, of object positions $\mathbf{u}$ onto the subspace of rigid-body object motions im $\left(\Gamma_{\mathrm{uc}}\right)$ :

$$
\begin{aligned}
& \mathbf{e}_{\mathbf{u c}}=\mathbf{E}_{\mathbf{u c}} \mathbf{x} ; \quad \text { where } \mathbf{E}_{\mathbf{u c}}=\Gamma_{\mathbf{u c}}^{\mathbf{P}}\left[\begin{array}{llll}
\mathbf{0} & \mathbf{I} & \mathbf{0} & \mathbf{0}
\end{array}\right] \\
& \text { and } \Gamma_{u c}^{P}=\Gamma_{u c}\left(\Gamma_{u c}^{T} \Gamma_{u c}\right)^{-1} \Gamma_{u c}^{T} .
\end{aligned}
$$

\section{Control of internal forces: a case study}

The following example shows the behaviour of an internal force controller designed for rigid-body systems but acting on a manipulation system with a deformable object. The planar manipulation system is the two Dof's depicted in fig. 2-a). It has the contact points in ${ }^{1}(0,2)$ and $(2,2)$, the prismatic joint in $(2,1)$, and stiffness, damping and inertia matrices normalized to the identity matrix. The Jacobian and the grasp matrix take the following values

$$
\mathbf{J}=\left[\begin{array}{cc}
-2 & 0 \\
0 & 0 \\
-2 & -1 \\
2 & 0
\end{array}\right] ; \mathbf{G}=\left[\begin{array}{cccc}
1 & 0 & 1 & 0 \\
0 & 1 & 0 & 1 \\
0 & -1 & 0 & 1
\end{array}\right]
$$

Observe that the system is defective, $\operatorname{ker}\left(\mathrm{J}^{\mathbf{T}}\right) \neq \emptyset$. The controlled output is $\mathbf{e}_{\mathbf{t i}}(2)$ : the projection of the force vector $\mathbf{t}$ onto the null space of $\mathbf{G}$, i.e. the subspace of reachable internal forces which, in this case, is $\mathcal{R}_{t i, \tau}=$ im ([1 $00-10])$.

The force control block diagram is reported in fig. 4 . It

\footnotetext{
${ }^{1}$ With respect to the depicted reference frame.
} 


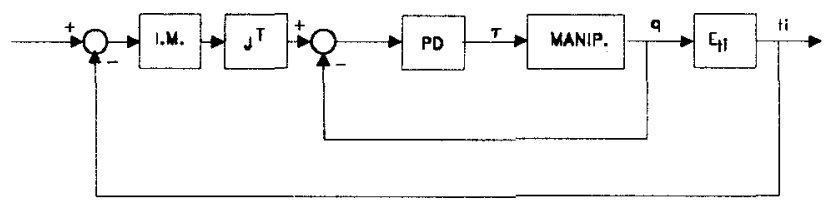

Figure 4: Two-loops internal force control law.

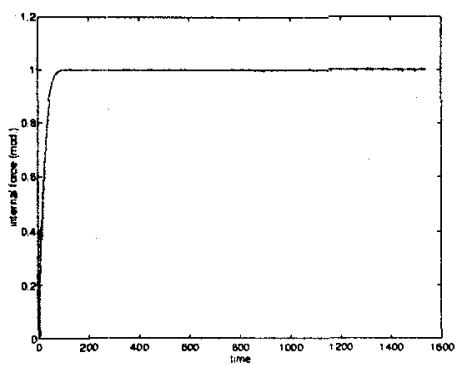

Figure 5: Simulation result of block diagram 4. Reachable internal force.

consists of two loops: the internal one is the stabilizing [7] PD controller of joint positions while the external one is the force loop composed by the internal model and the Jacobian transpose.

The internal model principle guarantees that when the input is a unit step the magnitude of the output $\mathbf{e}_{\mathbf{t} \mathbf{i}}$ reaches the unit value.

Unfortunately the controller action moves the grasped object along the rigid body coordinate subspace,

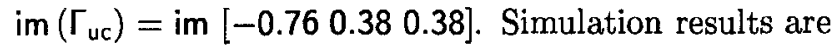
reported in fig. 5 and 6. The final configuration of the manipulation system looks like the one depicted in fig. 2-b). Observe that this is a dangerous maneuver in applications like those described in Section 1.

The paper contribution consists in proposing a state-

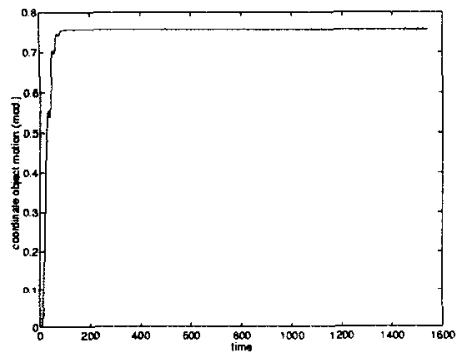

Figure 6: Simulation result of block diagram 4. Coordinate rigid-body object positions. feedback internal force controller whose action results to be decoupled from the rigid-body object dynamics.

\section{Object-motion decoupling control of internal forces}

This section is aimed at the analysis of the objectmotion decoupling control of internal forces for general grasping mechanisms. It should be underlined that, according to [8], by the term "general" we mean manipulation systems with any $\mathbf{J}$ and $\mathbf{G}$ with $\operatorname{ker}\left(\mathbf{G}^{\mathbf{T}}\right) \neq \mathbf{0}$ (no free object motion)a and that the manipulation system with kinematically deficiency $\left(\operatorname{ker}\left(\mathrm{J}^{\top}\right) \neq 0\right)$ is a paradigm of general manipulation systems.

Definition 1 Consider the dynamic system (1) A control law of the internal forces $\mathbf{e}_{\mathbf{t}}$ is decoupled with respect to the coordinate rigid-body motions $\mathbf{e}_{\mathrm{uc}}$, if there exists a linear combination $\mathbf{B}_{\tau} \mathbf{U}$ of the input-matrix columns such that, for zero initial condition, the input $\tau=\mathbf{U}_{\tau_{\mathbf{n}}}$ only affects the internal forces $\mathbf{e}_{\mathbf{t i}}$ while $\mathbf{e}_{\mathbf{u c}}$ remains identically zero.

The following theorem shows that this definition can be considered a structural property of general (nonindeterminate) manipulation systems.

Assume that:

H1: The manipulation mechanism is not indeterminate, i.e. $\operatorname{ker}\left(\mathbf{G}^{\mathbf{T}}\right)=\mathbf{0}$;

H2: $\operatorname{ker}\left(\Gamma_{\mathrm{uc}}^{\top}\right)$ is $\mathbf{M}_{\mathbf{0}}^{-\mathbf{1}} \mathbf{G K G}^{\mathbf{T}}$-invariant

Then the following theorem holds.

Theorem 1 Consider the linearized manipulation system (1). Under the hypothesis $\mathbf{H 1}-\mathrm{H2}$, there exists a stabilizing state feedback $\mathbf{F}$ and a matrix $\mathbf{U}$ such that

- the decoupling condition of Definition 1 holds

- $\operatorname{im}\left(\mathbf{E}_{\mathbf{t i}}<\mathbf{A}+\mathbf{B}_{\tau} \mathbf{F} \mid \mathbf{B}_{\tau}>\right)=\operatorname{im}\left(\mathbf{E}_{\mathbf{t i}}\right)$.

being $\langle\mathbf{A} \mid \mathbf{B}\rangle=\left[\begin{array}{llll}\mathbf{B} \mathbf{A B ~} \mathbf{A}^{2} \mathbf{B} & \cdots & \mathbf{A}^{\mathbf{n}-1} \mathbf{B}\end{array}\right]$.

Remark 1 Hypothesis $\mathrm{H} 2$ is technically in nature and has been introduced in order to simplify the proof of the theorem.

Remark 2 Theorem 1 states both the decoupling property and the complete output reachability of internal forces $\mathcal{R}_{t i, \tau}(3)$.

Remark 3 If the sensor system of the robotic manipulator is not able to measure the object position and velocity, a state observer is needed in the control loop. This commonly occurs when robots are equipped with tactile sensors. If contact force and joint position sensors are chosen, the local state detectability from the measured outputs has been proved [8]. 


\section{Proof of the theorem and controller design}

The groundwork of the decoupling theorem is the geometric approach to the multivariable control design $[3,13]$. Next, we recall a basic concept of this approach. Consider the linear dynamic system $\left(\mathbf{A}, \mathbf{B}_{\tau}\right)$ with a given subspace of the state space, say $\mathcal{S}$ and let us introduce the notion of $\mathcal{S}$-constrained reachable subspace, $R_{\mathcal{S}}$ with the following question. How does the reachability subspace change if the state trajectories are constrained to belong to the given subspace $\mathcal{S}$ ? The wrong answer would be that $\mathcal{R}_{\mathcal{S}}$ is equal to im $\langle\mathbf{A}| \mathbf{B}_{\tau}>\cap \mathcal{S}$. Under the hypothesis of existence of a state-feedback matrix $\mathbf{F}$ such that (s.t.)

$$
\left(\mathbf{A}+\mathbf{B}_{\tau} \mathbf{F}\right) \mathcal{S} \subseteq \mathcal{S},
$$

the correct answer [3] is that

$$
\mathcal{R}_{\mathcal{S}}=\mathrm{im}\left\langle\mathbf{A}+\mathbf{B}_{\tau} \mathbf{F}\right| \mathbf{B}_{\tau} \mathbf{U}>\subseteq \mathcal{S}
$$

where the input selection matrix $\mathbf{U}$ is such that

$$
\operatorname{im}\left(\mathbf{B}_{\tau} \mathrm{U}\right)=\operatorname{im}\left(\mathbf{B}_{\tau}\right) \cap \mathcal{S} .
$$

According to the theorem statement, it's now clear that to decouple the system, this should be controlled by constraining state trajectories to lie onto the null space of the rigid-body output matrix $\mathbf{E}_{\mathbf{u c}}$ (5).

Let us define the subspace $\mathcal{S}$ as

$$
\mathcal{S}=\operatorname{im}\left[\begin{array}{llll}
\mathbf{X} & \mathbf{0} & \mathbf{0} & \mathbf{0} \\
\mathbf{0} & \mathbf{0} & \mathbf{Y} & \mathbf{0} \\
\mathbf{0} & \mathbf{X} & \mathbf{0} & \mathbf{0} \\
\mathbf{0} & \mathbf{0} & \mathbf{0} & \mathbf{Y}
\end{array}\right]
$$

where $\mathbf{Y}$ is the maximum $\mathbf{M}_{o}^{-1} \mathbf{G} \mathbf{K G}^{\mathbf{T}}$-invariant subspace contained in $\operatorname{ker}\left(\Gamma_{\mathrm{uc}}^{\mathrm{T}}\right)$ :

$$
\mathbf{Y}=<\mathbf{G K G}^{\mathbf{T}} \mathbf{M}_{\mathbf{o}}^{-\mathbf{1}} \mid \Gamma_{\mathbf{u c}}>^{\perp}
$$

and $\mathbf{X}$ is s.t.

$$
\mathbf{M}_{o}^{-1} \mathbf{G K J X}=\mathbf{Y} \cap \mathbf{M}_{\mathrm{o}}^{-1} \mathbf{G K J},
$$

where notation is abused to indicate matrices and corresponding column spaces as well.

Being $\mathbf{Y}$ contained in $\operatorname{ker}\left(\Gamma_{\mathrm{uc}}^{\top}\right)$, from (5) it is an easy matter to verify that $\mathcal{S} \subseteq \operatorname{ker}\left(\mathbf{E}_{\mathbf{u c}}\right)$, thus, by choosing $\mathbf{U}$ as in (8) and $\mathbf{F}$ as in (6), according to (7) it follows that the state-feedback control system, depicted in fig. 7 and described by the couple $\left(\mathbf{A}+\mathbf{B}_{\tau} \mathbf{F}, \mathbf{B}_{\tau} \mathbf{U}\right)$, does not affect the rigid-body object motions. In fact its reachable subspace is a subset of $\operatorname{ker}\left(\mathbf{E}_{\mathbf{u c}}\right)$ :

$$
\mathcal{R}_{\mathcal{S}}=\mathrm{im}\left\langle\mathbf{A}+\mathbf{B}_{\tau} \mathbf{F}\right| \mathbf{B}_{\tau} \mathbf{U}>\subseteq \operatorname{ker}\left(\mathbf{E}_{\mathbf{u c}}\right) .
$$

The existence of a stabilizing feedback matrix $\mathbf{F}$ satisfying condition (6) is guaranteed by the fact that $\mathcal{S}$ is an internally stabilizable $\left(\mathbf{A}, \mathbf{B}_{\tau}\right)$-controlled invariant [3] and that system (1) is stabilizable $[9,10]$.

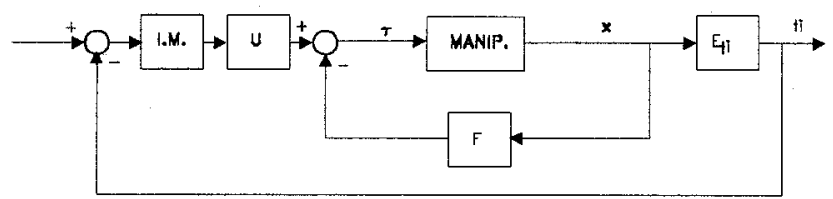

Figure 7: State-feedback decoupling controller.

After having decoupled the system outputs, it still remains to prove the rank condition stated in Theorem 1. The computation of the $\mathcal{S}$-constrained reachability subspace is needed. $\mathcal{R}_{\mathcal{S}}$ could be evaluated by using equation ( 7 ), the inconvenience is that this equation is a function of $\mathbf{F}$ which is not uniquely defined. A more convenient way to compute $\mathcal{R}_{\mathcal{S}}$ goes through the computation of the minimal conditioned and maximal controlled invariants, cf. [3] for more details. After some algebraic manipulations, we obtain that

$$
\mathcal{R}_{\mathcal{S}} \supseteq \operatorname{im}\left[\begin{array}{cccc}
\mathbf{X} & \mathbf{0} & \mathbf{0} & \mathbf{0} \\
\mathbf{0} & \mathbf{0} & \mathbf{P} & \mathbf{0} \\
\mathbf{0} & \mathbf{X} & \mathbf{0} & \mathbf{0} \\
\mathbf{M}_{\mathbf{0}}^{-1} \mathbf{G K J \mathbf { X }} & \mathbf{0} & \mathbf{0} & \mathbf{P}
\end{array}\right]
$$

where

$$
P=<M_{o}^{-1} G_{K G}{ }^{T} \mid M_{o}^{-1} G K J X>.
$$

In [10], it is proven that $\mathrm{im}(\mathbf{Q X})=\mathrm{im}(\mathbf{Q})$, consequently $\mathbf{E}_{\mathrm{ti}} \mathcal{R}_{\mathcal{S}}=\mathrm{im}\left(\mathbf{E}_{\mathrm{ti}}\right)$, and the theorem proof ends. Finally, the state-feedback control law, suggested by Theorem 1 and depicted in fig. 7, consists of a stabilizing feedback gain $\mathbf{F}$ which satisfies inclusion (6) and of the input selection matrix $\mathbf{U}$ defined by equation (8).

\section{Case study}

The case study has been introduced in Section 5 together with the non-decoupling control law of fig. 4 . Simulation results of Section 5 are here compared with those obtained by using the decoupling controller of Theorem 1.

According to the previous section, the state-feedback gain $\mathbf{F}$ and the input selection matrix $\mathrm{U}$ of the block diagram in fig. 7 are obtained as

$$
\mathbf{F}=\left[\begin{array}{cccccccccc}
5 & 2 & 4 & -4.5 & -4.5 & 4 & 2 & 4 & -4.5 & -4.5 \\
2 & -6 & 11 & 10 & 10 & 2 & -7 & 11 & 10 & 10
\end{array}\right]
$$

and

$$
\mathrm{U}=\left[\begin{array}{c}
-0.16 \\
0.99
\end{array}\right]
$$

The controlled output is $\mathbf{e}_{\mathbf{t} \mathbf{i}}$. Again the internal model principle guarantees that, with a unit step as input, the internal force $\mathbf{e}_{\mathbf{t}}$ reaches (in magnitude) the unit, but unlike the controller of fig. 4 , the decoupling control 


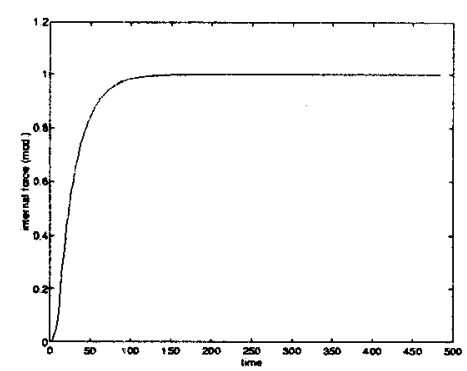

Figure 8: Simulation result of block diagram 7. Reachable internal force.

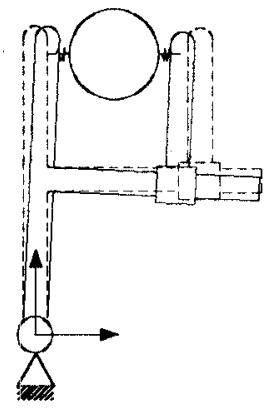

Figure 8: Decoupling internal force control.

of fig. 7 does not affect the rigid-body object motion which remains identically zero. In other words, the proposed internal force controller allows one to squeeze the object, see fig. 8, in a way that the manipulated object does not change its position. Fig.9 pictorially describes the squeezing action by the decoupling controller.

\section{Conclusions}

In this paper the problem of controlling internal forces of general manipulation systems is investigated.

Due to the presence of kinematically defective grasps as a relevant subclass of the general ones, the choice of the regulated output required particular attention. In power grasps, not all the contact forces are reachable.

Special attention was devoted to manipulation systems with significant compliance at the contacts. As a consequence, the object dynamics was taken into account in controlling the internal forces.

After characterizing the rigid-body object motions and the reachable contact forces, the problem of synthesizing an internal force control law which does not interact with the rigid-body object motion was investigated.

The geometric approach is used throughout the paper whose main result states that there always exists a state-feedback control law that decouples the internal force control action from the object dynamics. Note that such a decoupling property can be considered as a structural property of general manipulation systems.

\section{References}

[1] P.N. Akella and M.R. Cutkosky, "Contact transition control with semiactive soft fingertips," in $I E E E$ Trans. Robotics Automat., Vol. 11, No. 6, pp. 859-867. 1995.

[2] G. Basile and G. Marro "A state space approach to non-interacting controls, " Ricerche di Automatica, vol. 1, n. 1, pp. 68-77, 1970.

[3] G. Basile and G. Marro Controlled and conditioned invariants in linear system theory, New Jersey, Prentice Hall, 1992.

[4] A. Bicchi, "Force distribution in multiple wholelimb manipulation," in Proc. IEEE Int. Conf. Robotics Automat. pp. 196-201. 1993.

[5] A. Bicchi, C. Melchiorri and D. Balluchi, "On the mobility and manipulability of general multiple limb robots," IEEE Trans. on Robotics and Automat., vol. 11, n. 2, pp. 215-228, 1995.

[6] L. Meirovitch, Analytical methods in vibrations, Macmillan Pub. Co., Inc. New York. 1967.

[7] D. Prattichizzo, "Structural properties and control of robotics manipulation," $P h D$ Thesis, University of Pisa, 1995.

[8] D. Prattichizzo and A. Bicchi, "Dynamic analysis of mobility and graspability of general manipulation systems," in IEEE Trans. Robotics Automat. Accepted 1997. In press. Available from www.piaggio.ccii.unipi.it.

[9] D. Prattichizzo and A. Bicchi, "Consistent task specification for manipulation systems with general kinematics," in ASME Journal of Dynnamic System Measurements and Control. Accepted 1997. In press. Available from www.piaggio.ccii.unipi.it.

[10] D. Prattichizzo, P. Mercorelli, A. Bicchi and A. Vicino, "Noninteracting Force/Motion Control of Defective Manipulation Systems," in Proc. Int. Conf. Decision Control, pp. 1952-1957, 1996.

[11] J.K. Salisbury, "Whole-arm manipulation," in Proc. of the 4th International Symposium of Robotics Research, Cambridge, MA, MIT Press, 1987.

[12] J.C. Trinkle, "Dexterous manipulation planning and execution of an enveloped slippery workpiece," in Proc. IEEE Int. Conf. Robotics Automat., 1993.

[13] W.M. Wonham, Linear multivariable control: a geometric approach, New York, Springer-Verlag, 1979. 